\title{
De sujetos, redes y prácticas culturales
}

\author{
Ricardo A. Morales Lira
}

En las imágenes se funden la visión que la sociedad tiene de los jóvenes con, a veces, la que los jóvenes tienen de sí mismos y de la sociedad.

Giovanni Levy y Jean-Claude Schmitt

\section{Los sujetos}

C

ADA SOCIEDAD tiene su concepción de juventud a partir del ejercicio histórico y constante de construir múltiples formas de ser joven. Desde los efebos atenienses, los guerreros romanos, los nobles y plebeyos medievales hasta los punks, rastas, rockers y fresas de nuestros días (entre otras tipificaciones), las instituciones adultas iglesia, milicia, escuela, familia, sistemas jurídicos, etcéteraæ han puesto en marcha estrategias de configuración de mentalidades, culturas, sensibilidades, cuerpos, miradas, y que desde la óptica de la hegemonía consideran lo propio de y para este grupo.

Estas construcciones sociales descansan en proyectos políticos, económicos y culturales que se plantean como líneas prospectivas de lo que "queremos ser" como naciones, países, ciudades, barrios, familias, individuos.

Sin embargo, la juventud es una realidad terca, necia, no se deja atrapar ni mucho menos domesticar tan fácilmente, ella es plástica, movible, siempre en transición: las imágenes de la juventud no están cristalizadas, no son fijas, se parecen más a la gramática visual del cine, del performance. Por ello la multiplicidad de realidades hechas complejidad subjetiva en los jóvenes se mueve entre las antinomias de lo tradicional/moderno, reaccionario/revolucionario, conservador/liberal, místico/incrédulo, etcétera.

1. Universidad Iberoamericana, Tijuana. 
Las perspectivas que ven a estos sujetos como algo inacabado, todo y nada a la vez, indefinidos por naturaleza, que prueban de la vida lo que les va llegando, impiden observar estas características como elementos indispensables de la riqueza de cualquier cultura - no solamente las juveniles-, y permite continuar pensando a los jóvenes como una especie de rompecabezas sin identidad: mirarlos así apuntala una perspectiva unidimensional. Toda cultura, como sistema complejo de construcciones simbólicas y realizaciones materiales, es contradictoria, paradojal.

Recordemos: los jóvenes siempre han sido sujetos de impugnación, de reclamo, pero también generadores de propuestas políticas y culturales - sobre todo desde los 50s del siglo XX - manifestadas en expresiones concretas que van desde la música, los movimientos gays y feministas, pasando por el revival del misticismo, las religiones milenarias y la aparición de nuevas; el new age, etcétera; hasta el activismo de jóvenes intelectuales luchando por sus reivindicaciones universitarias, artísticas y ciudadanas. La emergencia de otro mundo distinto al de mediados del siglo pasado ya se dio.

$\mathrm{Si}$ aceptamos la afirmación de que cada sociedad elabora sus nociones y proyectos sobre los jóvenes, también debemos reconocer la existencia de análisis serios y sistemáticos, mismos que inician un camino nuevo sobre el entendimiento de la juventud.

El camino va desde los primeros estudios sociológicos a principios del siglo XX con la escuela de Chicago, pasando por las reflexiones filosófico-políticas de Gramsci y los estudios de Parsons, hasta la nueva propuesta de una antropología de la juventud (Feixa, 1998: 9). Así, la investigación sobre juventud ha girado en tres vertientes fundamentales, a saber.

Por un lado, los primcros estudios, abocados a las relaciones sociales patológicas y anómicas de los jóvenes en el entorno urbano; esto es, el pandillerismo, la drogadicción, las bandas o "gangs", entre otras agrupaciones. Estos trabajos se centraron en lo que se consideraban conductas desviadas de adolescentes y jóvenes en la urbanidad.

Por otro, las reflexiones sobre las posibilidades de que los jóvenes a futuro se convertirían en guías de carácter moral, intelectual, político y cultural, mismos que harían posible la revolución al interior de sus respectivos estados/naciones: éstos son los intelectuales orgánicos gramscianos.

Por último, y más recientemente ædesde mitades de los 80 s. aparecen estudios dedicados a comprender las dinámicas culturales, políticas e históricas que revisten las llamadas culturas juveniles.

Desde el filón gramsciano donde se analizan las relaciones entre la cultura hegemónica y las subalternas, así como las divergentes mediaciones que existen entre estos dos niveles, trabajos como los de José Manuel Valenzuela (1988) y los de Carlos Feixa (1998), abren brecha al integrar el concepto de culturas juveniles en plural, pues para ambos autores lo que se le ha denominado cultura juvenil, en singular, no da 
cuenta de la complejidad de las distintas, heterogéneas y múltiples experiencias sociales de los jóvenes, pues "sabemos que en la complejidad de nuestras sociedades, el verbo "cultura" se conjuga siempre en plural: cultura(s) múltiples, diversas, desniveladas, rejegas, modernas, arrítmicas, sincopadas, que a su modo resuellan, pujan, jadean y se jalonean entre sí, sin tregua duradera y a veces con particulares tonalidades cáusticas y cuantimás conflictivas." (González; 1994, 9-10)

Así, definir lo juvenil no ha sido fácil. Conceptos como identidad/alteridad, modernidad, posmodernidad, globalización, regionalización, desterritorializaciónterritorialización, nuevas sensibilidades (Cfr. Martín-Barbero; 1997), entre otras acepciones, surgen ahora como elementos contextuales en la comprensión de lo juvenil.

Asimismo, ante las visiones de que estas culturas, como fenómeno social, son algo dado, hay respuestas como las de José Manuel Valenzuela quien en contra de esta tendencia positivista afirma que "lo dado era su inexistencia; lo que existía eran unas formas de expresión cultural juveniles diferenciadas y que era difícil hablar de la cultura juvenil en ámbitos tan heterogéneos”. (Valenzuela, 1998: 11)

Pese al enorme esfuerzo por entender las experiencias juveniles, muchos de estos últimos trabajos fijan su atención en las llamadas manifestaciones de jóvenes culturalmente visibles ( $C f r$. Rosaldo; 1991) y con estilos espectaculares propios ( $C f r$. Feixa, 1998: 97): chavos banda, cholos, rockeros, punks, etcétera.

Sin embargo, aquellos sujetos que se nos aparecen como gente sin cultura, como personas comunes y corrientes que no intentan "verse culturalmente", pero manifiestan una incesante $y$, a menudo, inadvertida producción cultural, no les hemos puesto la debida atención. Estos sujetos no están culturalmente desprovistos; al contrario poseen prácticas culturales propias que no entran en las categorías de lo marginal o subalterno.

Por lo anterior, el trabajo de tesis no gira en torno a las culturas subalternas, marginales, etcétera, sino a aquellas prácticas culturales que los jóvenes establecen al interior de espacios concretos de producción simbólica, como la escuela, la iglesia, la familia, los espacios de diversión; prácticas vividas en la cotidianidad, difíciles de esclarecer, pero exigen investigación y análisis

Por ello la problemática aquí tratada es la construcción social de los jóvenes, pero no desde las estrategias del mundo adulto para moldearlos culturalmente, y de cómo los primeros participan también (de forma activa) en esa construcción social. El énfasis del trabajo está en las formas de producción cultural generadas, producidas y puestas en circulación por los jóvenes mismos.

En otras palabras, pretendemos comprender las redes de comunicación tejidas socialmente por los jóvenes. Asimismo, intentamos explorar las distintas prácticas culturales realizadas por ellos al interior de espacios concretos de producción simbólica, así como el consumo de determinadas ofertas culturales. 


\section{Las preguntas-guías de investigación}

Nuestros entornos urbanos son espacios sociales de interacción y significación que permiten la existencia de sujetos complejos. La diferenciación y multiplicidad de estos espacios componen lo que Maffesoli denomina socialidad, es decir, la estructuración del tiempo/espacio donde los individuos æcomponentes aislados de lo socialæ, pasan a ser personas æelementos estructurados en relaciones socialesæ, esto es a "hombres en relación; y no sólo a la relación interindividual, sino también a eso que me liga a un territorio, a una ciudad, aun entorno natural, que yo comparto con otros." (Maffesoli; 1990, 213-214)

Los espacios sociales siempre están cargados de significación, son lugares culturalmente practicados; es decir, son antropológicos por excelencia.

En este sentido, las prácticas culturales y las redes que los jóvenes construyen en dichos espacios de socialidad permiten una serie de interacciones simbólicas, de vínculos afectivos, cognitivos, de contactos reales y virtuales, de configuración de comunidades de sentido que forman gran parte de la constitución subjetiva de estos actores, pues de hecho "la organización de la socialidad tiene forma de red. Una red que conecta nudos”. (Ibáñez, 1990: 16)

En otras palabras, la multiplicidad de dichas prácticas imbricadas con el consumo de determinadas ofertas culturales y las redes de comunicación de los jóvenes, procuran una ecología propicia para la construcción de estilos de vida y experiencias sociales juveniles, denominadas culturas juveniles; éstas se "refieren a la manera en que las experiencias sociales de los jóvenes son expresadas colectivamente mediante la construcción de estilos de vida distintivos, localizados fundamentalmente en el tiempo libre, o en espacios intersticiales de la vida institucional". (Feixa, 1998: 84)

Por lo anterior, las preguntas que guían nuestro trabajo de investigación son las siguientes:

¿Cómo se han generado redes de comunicación entre los jóvenes? ¿Cuál es la composición de dichas redes?

¿Qué tipo de información generan y cómo son sus prácticas de comunicación?

¿Cuáles son los campos de cultura que se cruzan con las distintas prácticas cotidianas de estos sujetos, así como el consumo de las ofertas culturales de estos campos, procurando lugares de contacto e interacción, así como discursos y creaciones que ellos producen y circulan en espacios concretos?

¿A lo largo de sus vidas, cómo han sido y son sus experiencias sociales y la forma de percibir "lo joven", sobre todo en el marco de las porosas fronteras culturales y espacios intersticiales? 


\section{Redes, prácticas culturales y percepciones de lo juvenil: una primera mirada}

Preguntar por el otro una y otra vez. Indagar su actuar, su estar en el mundo. Escudriñar sus miradas, admirar la manera como éstas se moldean junto con los cuerpos. Rastrear las improntas, las huellas que hombres y mujeres dejan a través de sus rutas, viajes, navegaciones que se convierten en índices a ser leídos. Sorprendernos ante la magia de las sensibilidades cambiantes, profundas, metamorfoseadas, barrocas. Leer en ellas nuestra memoria, los proyectos vitales e históricos que hacen de la cotidianidad nuestra pequeña ínsula, nuestra gran aventura.

Y a todo eso nombrarlo, darle sentido, recrearlo a través del lenguaje, pues la crisis de lo social pone en igual situación a las actuales categorías de comprensión que piden su reactualización ante la emergencia -en estos momentos de transicionesde nuevos sujetos, miradas recientes, otros cuerpos, órdenes nuevos; a pesar de que, como afirma James Clifford, "es más fácil registrar la pérdida de órdenes tradicionales de diferencia que percibir la emergencia de nuevos" (Clifford, 1995: 31).

Precisamente, pensar en las nuevas realidades es hacer preguntas distintas a las tradicionales. No obstante, las viejas realidades nutren de vivacidad a la actuales, muestran hitos históricos a perseguir, pues para entender el futuro es imprescindible la reinterpretación del pasado (Le Goff, 1991). "Generar lo nuevo - no sólo como algo antes inexistente, sino también como relectura de nuestra historia y de nuestra memoria, de nuestros proyectos, débiles, fuertes o desmesurados, desde nuestra relación corporal y social con el territorio y la gente-, acompañarlo, implica en este caso no proponer un orden o una fórmula". (Ford, 1994; 55).

Es entonces, el momento de comenzar a indagar acerca los nuevos sujetos e identidades, sobre sus redes de relaciones y lo que culturalmente crean; sobre la construcción de sensibilidades jóvenes con características hasta hace poco inexistentes, mismas que hoy tienen un papel protagónico en los escenarios sociales.

Así las cosas, en la tesis intentamos investigar las redes sociales que permiten la construcción de sentidos, redes generadas por los jóvenes dentro de sus entornos urbanos. Así este capítulo está armado por ideas/conceptos que funcionan como punto de arranque para cuestionarnos acerca de las nuevas configuraciones de relaciones entre los actores-red ubicadas justo en el lugar "natural" de su existencia: la estructura social (Arvanitis, 1996). Estructura mediada por las prácticas cotidianas de comunicación y cultura en íntima relación con los espacios de producción sígnica institucional.

Espacios en los cuales nuestras sociedades contemporáneas han generado una serie de instituciones especializadas en la producción discursiva y de bienes simbólicos, llamados campos culturales (Bourdieu, 1986), en íntima relación con el con- 
sumo de las ofertas de estos campos. Entre otras cosas, lo anterior ha permitido diferentes formas simbólicas y maneras distintas de percibir el mundo y actuar en él. Aquí el concepto de consumo cultural es importante, pues nos permite "mirar" teóricamente ciertos procesos y relaciones sociales de los jóvenes, ya que éste es "el conjunto de procesos de apropiación y usos de productos en los que el valor simbólico prevalece sobre los valores de uso y de cambio, o donde al menos estos últimos se configuran subordinados a la dimensión simbólica" (García Canclini, 1993: 34).

Los jóvenes son sujetos sociales que elaboran redes de comunicación entretejidas con prácticas de cultura múltiples y diversificadas, es decir, son creadores de innovadoras relaciones sociales mediadas por los distintos campos culturales.

La imbricación entre el consumo de las ofertas culturales, los usos específicos del espacio urbano marcados por la vida cotidiana y las microculturas familiares (Cfr. Bertaux y Bertaux-Wiame, 1994: 27) (González Sánchez, 1995), hacen posible la configuración de habitus, esto es, todo "un sistema de esquemas adquiridos que funcionan en estado práctico como categorías de percepción y de apreciación o como principios de clasificación al mismo tiempo que como principios organizadores de la acción" (Bordieu, 1988: 26).

Por ello, los jóvenes realizan prácticas comunicativas, creaciones que tienen que ver con todo aquello que han interiorizado a lo largo de sus vidas. Así, la comunicación como proceso de múltiples y complejas formas simbólicas y materiales de la cultura, opera en sus pequeños mundos, esto último es la vida cotidiana.

Por lo anterior el trabajo se centra en indagar las experiencias de vida y la percepción de los jóvenes, así como sus redes de comunicación, en íntima relación con espacios concretos de prácticas culturales hechas por ellos.

Trabajemos los conceptos.

Las redes son sistemas complejos compuestos por vínculos, relaciones, contactos, formas de comunicación. Tomás Rodríguez Villasante (1998) plantea una imagen donde cada sujeto es un nodo y la intensidad de relación entre los sujetos produce "nodos de resonancia", esto es, vínculos de comunicación.

Asimismo, dichas redes están tejidas por prácticas culturales que los jóvenes ponen en juego. Mismas que han estado cambiando en la medida que los distintos sujetos han podido generar relaciones sociales más horizontales, participativas y generadoras de información.

Estos sujetos, asimismo, construyen comunidades de sentido, lugares de encuentro donde hay elementos simbólicos en común que dan coherencia interna a las redes.

A pesar de que nuestras redes sociales existen previamente a nuestra vida (muchas de ellas son heredadas, impuestas), a pesar de que las habitamos y, a menudo no las vemos, no las racionalizamos ni explicitamos, pero sí las vivimos, las sentimos incesantemente; los jóvenes también elaboran redes complejas de comu- 
nicación desde las cuales realizan una serie productos culturales estrechamente ligados al consumo de distintas ofertas de cultura y a la cotidianidad en el espacio urbano, lugar privilegiado, este último, en el que se manifiestan expresiones juveniles como la música, el deporte, la diversión y la educación, entre otros ámbitos.

La red, "como modelo de acción humana" (Arvanitis, 1996: 44) es a la vez un modelo de comprensión, y espacio social de existencia, de interacción y vínculos, pero asimismo es un concepto que nos describe relaciones, mismas que tienen como característica fundamental el flujo de información que circule en todas direcciones. La participación de los sujetos-red en la elaboración y uso de la información tiende a ser colectiva. Por eso, las redes se parecen más a sistemas de comunicación con una altísima cultura de información (Galindo, 1996), misma que procura prácticas, creaciones y expresiones, las cuales no se oponen a las de espacios institucionales.

Más aún. Las redes, como estructuras de organización social, están compuestas de lógicas distintas a los espacios formales y rígidos. Sus pautas de acción son flexibles como para que cada sujeto participe con lo que pueda aportar, pero siempre conservando el compromiso de hacer algo conjuntamente con los demás miembros de la red. Esta apertura reticular se funda en la solidaridad, el afecto, el respeto y la comunicación en todos y hacia todos los ámbitos.

Es necesaria, pues, la existencia de redes que crucen, alimenten, refresquen las instituciones, las universidades, las fronteras entre lo público y lo privado; redes que formen parte de nuestra cotidianidad, donde amigos, compañeros pares, partiendo de objetivos comunes podamos seguir construyendo estas redes "para mejor-vivir" (Rodríguez Villasante; 1998).

Gran parte del mundo juvenil está constituido por esas redes que tienen su existencia en el aquí y el ahora pero que están engarzadas con sus propias rutas de vida y de familia, por sus historias personales, familiares y sociales. En este orden de ideas podemos definir a la juventud como una construcción social históricamente determinada donde coexisten múltiples relaciones de poder. En palabras de Bourdieu:

Esta estructura, que existe en otros casos (como en las relaciones entre los sexos), recuerda que en la división lógica entre jóvenes y viejos está la cuestión del poder, de la división (en el sentido de repartición) de los poderes. Las clasificaciones por edad (y también por sexo, o, claro, por clase...) vienen a ser siempre una forma de imponer límites, de producir un orden en el cual cada quien debe mantenerse, donde cada quien debe ocupar su lugar (Bourdieu, 1990, 164).

La juventud como una estructura relacional, es decir, como todo un sistema complejo de elementos que van desde las acciones, las valoraciones y percepciones hasta las formas simbólicas que dan cabida a sistemas reticulares de un orden de complejidad mayor.

Pues como formas simbólicas las redes no sólo nos permiten ecologías sígnicas 
e interpretativas, sino también pautas cognitivas que nos guían en las acciones sociales, pues toda forma es:

un orden de relaciones inteligibles frente a las cuales el sujeto adopta tendencias y procesos rítmicos, condicionantes en primer lugar de su experiencia y que posteriormente pueden ser condicionantes de su entender ciertas relaciones, de sus modos de verificación y emisión de juicios, de sus modos de deliberar sobre la conveniencia de cada una de sus posiciones y de sus decisiones (Paoli, 1999: 41).

\section{La estrategia metodológica}

Cómo hacer observable nuestro objeto de estudio. En sentido metafórico: qué tipos de caminos (métodos) y maneras concretas de caminar (técnicas) estamos eligiendo y por qué. En términos más académicos: qué formas de intervención exige nuestro objeto de estudio para poder aprehenderlo y dar cuenta de él. Es decir, qué tipo de tecnologías de investigación usamos, para qué y por qué. ${ }^{2}$

Toda elección metodológica no es neutral, comporta toda una serie de elementos que determinan el uso de tal o cual método y su(s) correspondiente(s) técnica(s).

Dentro de estos elementos juega un papel importante la experiencia del investigador: su edad, el género, su formación académica y muchos otros factores que ponen por delante la subjetividad, sobre todo en las metodologías cualitativas.

Pero la cuestión también tiene que ver con el dominio de ciertos paquetes metodológicos; es decir, el conjunto de múltiples elecciones, decisiones e intervenciones sobre nuestro objeto de estudio mediante la igual multiplicidad de técnicas de investigación, lo que Jorge González llama polifonía metodológica. $(1993,225)$

Por ello, los objetos cognitivos son observar, describir y comprender las redes de comunicación, las prácticas culturales y la percepción que los jóvenes tienen de sí mismos.

Para lograr lo anterior decidimos trabajar con la etnografía. La etnografía focaliza su atención en las situaciones sociales configuradas por los sujetos, intenta realizar grandes catálogos de sujetos, acciones y objetos inscritos en un espacio/ tiempo determinado.

Así, por una parte se trató de observar, describir y comprender la composición de los espacios sociales al interior de los cuales los jóvenes realizan, en distintos ritmos, sus prácticas y creaciones culturales. Siguiendo a Spradley (1980), ubicamos la mirada en lo que los jóvenes hacen (cultural behavior), conocen (cultural

2. Entendemos por tecnologías de investigación las formas reflexivas y constructivas de usar determinados métodos y sus técnicas de investigación. Cfr. Jesús Galindo: http://www.geocities.com/ diplotecnicas/diplomado.htm 
kwowledege), usan y crean culturalmente (cultural artifacts).

Concretando lo anterior, se trabajó con:

- La composición social de espacios donde los jóvenes ponen en juego sus redes de comunicación y realizan sus prácticas culturales.

- Descripción tipológica de los sujetos en esos espacios.

- El lenguaje propio de los jóvenes y sus formas comunicativas.

- Los saberes cotidianos de los jóvenes.

- Sus objetos y creaciones culturales.

Con la observación participante se quiso desentrañar la importancia de la composición social de lugares donde los jóvenes construyen sus redes de comunicación y sus prácticas culturales: concretamente en espacios de diversión, educación y de comunicación.

Sin embargo, abordar un objeto de estudio multidimensional como el propuesto aquí, implica no solamente una mirada teórica igualmente compleja, sino tenemos que intentar una aproximación holística desde lo que denominamos sistemas de información metodológico y tecnológico.

También se realizaron entrevistas cualitativas para generar relatos orales en tres niveles. La importancia de esta técnica, generalmente perteneciente a los métodos de la historia oral, nos permite reconstruir, desde la perspectiva del sujeto, la experiencia vital y social, las rutas biográficas, la percepción del mundo de los sujetos y los referentes empíricos que han hecho posible que los jóvenes construyan su mundo de cierta manera y no de otra. Asimismo, una parte de las entrevistas permitirá ver las formas de consumo cultural (relación con los campos propuestos abajo) de los jóvenes, así como la importancia de sus microclimas culturales (Bertaux; 1994), propios de la familia y los grupos de amigos.

Precisamos los niveles de los relatos:

- RELATO LIBRE: podemos hablar de un Relato Biográfico; esto es la construcción narrativa biográfica del sujeto, para rastrear los momentos donde nuestro informante contacta con la música, la diversión, la educación, etcétera, así como las influencias de sus espacios privados de convivencia. Esta parte es semejante a la historia de vida.

- RELATO EXPLORATORIO: aquí trabajamos con temáticas, teóricamente consideradas campos culturales en la perspectiva de Bourdieu, ${ }^{3}$ mismos que creemos importantes en la vida de los jóvenes.

3. Cfr. Bourdieu, Pierre (1986). Cosas dichas, Gedisa, Barcelona. 
(CAMPOS)

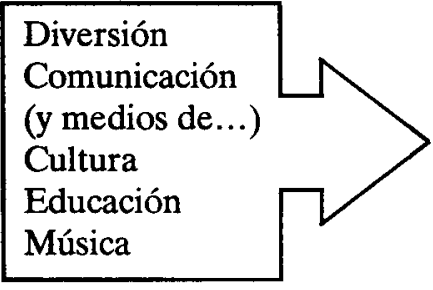

Nota: Aquí trabajamos con las rutas sociales, es decir, con los momentos mediante los cuales los jóvenes traban contacto con estos campos y sus respectivas ofertas culturales.

- RELATO PROFUNDO: en este nivel se exploró a profundidad los elementos que de los dos momentos anteriores se desprendan. Por ejemplo, en tres entrevistas realizadas, la familia resalta como elemento principal en su discurso. Allí trabajamos a profundidad. Para esta parte, no hay preguntas cerradas, pues aunque cerremos para explorar temáticas o elementos concretos, volvemos a abrir para ahondar en ellas.

A la par de la observación participante y la entrevista, usamos la metodología de Redes, misma que es un conjunto de instrumentos potentísimos para poder entender las estructuras relacionales y reticulares de los sujetos.

Independientemente de que al análisis de redes - o network analysis como se le conoce en la versión sajona - se le considera a la vez una metodología, un conjunto de técnicas, un sistema teórico y un paradigma científico; nuestras pretensiones en este trabajo son más humildes. Lo que intentamos con este sistema metodológico fue "radiografiar" (si se nos permite el término) mediante el trazado de redes, las relaciones sociales de jóvenes universitarios que incursionan en los ámbitos de la investigación y la creación.

Asimismo, trabajamos con dos grupos de discusión: uno de hombres y otro de mujeres. Con ellos intentamos "determinar formaciones discursivas colectivamente construidas" (González, 1998: 161) por jóvenes fronterizos en relación con su vida cotidiana. Aquí se trató de recuperar, mediante la reconstrucción del discurso grupal social, las percepciones que éstos sujetos tienen sobre si mismos, la cultura en la frontera y su relación con la ciudad y el consumo en los Estados Unidos, concretamente en San Diego, California.

El Grupo de Discusión, como tecnología de investigación, es un conjunto de recursos para el registro de las percepciones, apreciaciones, valoraciones y representaciones sociales de los sujetos (Ibánez, 1986).

El grupo de discusión es una técnica de investigación social que (como la entrevista abierta o en profundidad, y las historias de vida) trabaja con el habla. En ella, lo que se dice ælo que alguien dice en determinadas condiciones de enunciaciónæ, se asume como punto crítico en el que lo social se reproduce y cambia, como el objeto, en suma, de las 
ciencias sociales. En toda habla se articula el orden social y la subjetividad" (Canales y Peinado, 1995: 298).

Juntar un grupo para que los participantes se pongan a dialogar entre ellos no es tarea fácil: el reclutamiento de las personas, la disposición de tiempo por parte de ellas para la sesión, contar con el lugar adecuado para realizar ésta; todos estos elementos, entre otros muchos más, aparentemente circunstanciales en la puesta en marcha de la técnica, son determinantes en el logro o fracaso de las sesiones. Estas cuestiones no expuestas en los manuales de técnicas de investigación, son claves para que un grupo de discusión se concrete y no "se caiga", dicho en la jerga utilizada en esta técnica. La investigación, por ello, se aprende haciéndola, no nada más en recetarios de metodologías y técnicas.

Así las cosas, observación participante, entrevistas de historia oral, análisis de redes y grupos de discusión son nuestras tecnologías de investigación, mismas que permiten, desde una perspectiva multimetodológica, dar cuenta de esta realidad tan compleja llamada redes de jóvenes.

\section{La elección de los sujetos de estudio y la metodología cualitativa}

Como habíamos mencionado en líneas anteriores, para un objeto de estudio múltiple y complejo, es necesario el uso de una metodología compleja. Lo anterior se fundamenta en la razón de que para dar cuenta de un fenómeno social tenemos abordarlo que desde diversas perspectivas.

Entre otras cosas, lo anterior determinó las unidades de observación. Por ello, se decidió trabajar con jóvenes entre 18 y 22 años. Hombres y mujeres que estudien en la universidad. ¿Por qué esa edad y por qué las categorías de estudiantes?

La razón se fundamenta en la propuesta de Pierre Bourdieu (1994), quien afirma que a la juventud no se le puede clasificar por clases sociales sino por ocupación u oficio. Además creemos que entre los 18 y 22 años, los jóvenes comienzan a decidir sus espacios de estudio, ocio y trabajo, además, con cierta autonomía traban contacto con las ofertas culturales, así como sus elecciones, gustos y preferencias se ven más libres de la carga familiar o el mundo adulto.

Asimismo, esta razón tiene que ver con dos elementos importantes: el primero es el hecho de que sin tener "manifestaciones espectaculares" (Urtiaga, 1996), este tipo de jóvenes tienen creaciones y manifestaciones culturales riquísimas que piden investigación. Feixa afirma que "en general se ha tendido a menospreciar la diversidad de identidades juveniles presentes en México; el estudio de lo "marginal" se ha impuesto sobre el estudio de lo "normal" (tenemos muchos datos sobre drogas y violencia, pero pocos de familia, escuela, vida cotidiana); lo subalterno 
sobre lo hegemónico (los estudios sobre los chavos banda, pero no conozco uno sobre los chavos fresa); lo masculino sobre lo femenino (sabemos mucho sobre los machines pero poco de las quinceañeras); lo metropolitano sobre lo provinciano (conocemos muy poco sobre la identidad de los jóvenes indígenas, campesinos o de ciudades medias)" (Feixa; 1993a).

El segundo elemento es el hecho de que estos jóvenes tienen posibilidades de consumo cultural distintas a otros provenientes de otras clases sociales. Este consumo ha configurado nuevas sensibilidades y maneras distintas de percibir el mundo.

En palabras de Jesús Martín-Barbero:

Una generación que ha aprendido a hablar inglés en programas de televisión captados por antenas parabólicas, que se siente más a gusto escribiendo en el computador que en el papel, y tiene una empatía "natural" con la cultura tecnológica. Frente a la memoria larga pero también la rigidez de las identidades tradicionales los sujetos de la nueva generación parecen dotados de una plasticidad neuronal que se traduce en elasticidad cultural, una camaleónica capacidad de adaptación a los más diversos contextos, y una complicidad expresiva con el universo audiovisual e informático: en sus imaginerías y sonoridades, en sus fragmentaciones y velocidades, ellos encuentran su ritmo y su idioma (Martín-Barbero, 1997: 93-94).

En este sentido, si queremos reconstruir la experiencia cotidiana y la construcción social de los jóvenes a partir de sus redes, prácticas de cultura, así como el uso y apropiación de las ofertas culturales, la metodología cualitativa nos permitirá acceder a este mundo subjetivo y de las percepciones que tienen los jóvenes sobre sí mismos. En palabras de Taylor y Bogdam: "afirmamos que la década pasada fue testigo de un creciente interés por el lado subjetivo de la vida social, es decir, el modo en que las personas se ven a sí mismas y a su mundo. Escribimos entonces que este interés requería métodos descriptivos y holísticos: métodos cualitativos de investigación" $(1998,11)$.

Para finalizar este apartado, sólo quiero mencionar lo siguiente: nombrar lo escasamente nombrado, aquello que apenas se atisba, es un acto en el cual está más de por medio la conjetura, la lógica de la abducción o simplemente la corazonada, y de eso están compuestas las culturas, pues en tiempos de crisis e incertidumbre son ellas las que creativamente reaniman nuestro solemne mundo. Borges insistió: el día que se agoten nuestros reinos sólo nos quedará reinventarlos a través de los sueños, el amor y la palabra. 


\section{Referencias bibliográficas}

Arvantis, Rigas (1996). Redes de investigación e innovación: un breve recorrido conceptual, en Revista Latinoamericana de Estudios del Trabajo, año 2, núm. 3.

Bertaux, Daniel (1994a). Genealogías sociales comentadas y comparadas, en Estudios sobre las Culturas Contemporáneas, Vol. Vl, núm. 16-17, Programa Cultura, Universidad de Colima, Colima.

Bertaux, Daniel y Bertaux-Wiame, Isabel (1994). El patrimonio y su linaje. Transmisiones y movilidad social en cinco generaciones, en Estudios sobre las Culturas Contemporáneas Vol.Vl, núm. 18, Programa Cultura, Universidad de Colima, Colima. Bourdieu, Pierre (1986). Cosas dichas, Gedisa, Barcelona.

- (1987). Los Tres Estados del Capital Cultural, en Sociológica, año 2, núm. 5, UAM-Azcapotzalco, México.

- (1990). Sociología y cultura, Grijalbo-CNCA, México.

Canal, Manuel y Peinado, Anselmo (1995). Grupos de discusión, en Delgado, Juan Manuel y Gutiérrez, Juan, Métodos y técnicas cualitativas de investigación en ciencias sociales, Síntesis, Madrid.

Clifford, James (1995). Dilemas de la cultura. Antropología, literatura y arte en la perspectiva posmoderna, Gedisa, Barcelona.

Feixa, Carles (1993a). La ciudad en la antropología mexicana, Universidad de Lleida, España

- (1993b). De las bandas a las culturas juveniles, en Estudios sobre las Culturas Contemporáneas, Vol. V, núm. 15, Programa Cultura, Universidad de Colima, Colima, Col.

- (1998). De jóvenes, bandas y tribus. Antropología de la juventud, editorial Ariel, Barcelona.

Ford, Aníbal (1994). Navegaciones. Comunicación, cultura y crisis, Amorrortu, Argentina.

Galindo, Jesús (1996). Cultura de información, política y mundos posibles, en Estudios sobre las Culturas Contemporáneas, época II, volumen II, núm. 3, Programa Cultura, Universidad de Colima, Colima.

García Canclini, Néstor. (coord.) (1993). El consumo cultural en México. CNCA, México

Goffman, Irving (1986). La presentación de la persona en la vida cotidiana, Amorrortu editores, Buenos Aires.

González S. Jorge (1993). Metodología y Sociología Reflexivas. Navegar procelosos mares de placer, en Estudios sobre las Culturas Contemporáneas, Vol. V, núm. 15, Programa Cultura, Universidad de Colima, Colima, Col.

- (1994). La transformación de las ofertas culturales y sus públicos en México en Estudios sobre las Culturas Contemporáneas, Vol. VI, núm. 18, Programa Cultura, Universidad de Colima, Colima, Col. 
- (1995). Y todo queda entre familia: estrategias, objetos y método para historias de familia, en Estudios sobre las culturas contemporáneas, época II, Vol. 1, núm. 1, Programa Cultura Universidad de Colima, Colima, Col.

Ibáñez, Jesús (1990). Prólogo a El tiempo de las tribus, de Michel Maffesoli, Icaria, España.

Legoff, Jacques (1991). Pensar la historia. Modernidad, pasado y presente, Paidós, Barcelona.

Levi, Giovanni y Schmitt, Jean-Claude (1996) (coord.) Historia de los jóvenes, 2 tomos, Taurus, Madrid.

Maffesoli, Michel (1990). El tiempo de las tribus, Icaria, España.

Martín-Barbero, Jesús (1997). Descentramiento cultural y palimpsestos de identidad, en Estudios sobre las Culturas Contemporáneas, época II, volumen III, núm. 5, Programa Cultura, Universidad de Colima, Colima, Col.

Morales Lira, Ricardo y García Cortez, Alfonso (1995). La Revolución también es una calle. De frentes, fronteras y cruces culturales, en Estudios sobre las Culturas Contemporáneas, época II, vol. I, núm. 2, Programa Cultura, Universidad de Colima, Colima.

Rodríguez Villasante, Tomás (1998). Cuatro redes para mejor-vivir, 2 tomos, Editorial Lumen-Humanitas, Argentina

Rosaldo, Renato (1991). Cultura y verdad, Grijalbo-CNCA, México.

Spradley, James P. (1980). Participant Observation, Holt, Rinehart and Winston, USA.

Taylor, S. J. y Bogdam, R. (1998). Introducción a los métodos cualitativos de investigación, Paidós, Barcelona.

Urteaga, Maritza (1996). Organización juvenil. En Jóvenes: una evaluación del conocimiento. La investigación sobre juventud en México 1986-1996. Tomo II, Causa Joven, México.

Valenzuela Arce, José Manuel (1988). iA la brava, ese!, COLEF, México.

- (1998) Las producciones culturales y el consumo cultural, en Padilla Herrera, Jaime Arturo, (comp.), La construcción de lo juvenil, Causa Joven, México. 